\title{
On the blow-up solutions for the nonlinear Schrödinger equation with combined power-type nonlinearities
}

\author{
Binhua Feng \\ Department of Mathematics, Northwest Normal University, Lanzhou, 730070, P.R. China \\ $\llbracket$
}

\begin{abstract}
This paper is devoted to the analysis of blow-up solutions for the nonlinear Schrödinger equation with combined power-type nonlinearities

$$
i u_{t}+\Delta u=\lambda_{1}|u|^{p_{1}} u+\lambda_{2}|u|^{p_{2}} u .
$$

When $p_{1}=\frac{4}{N}$ and $0<p_{2}<\frac{4}{N}$, we prove the existence of blow-up solutions and find the sharp threshold mass of blow-up and global existence for this equation. This is a complement to the result of Tao et al. (Comm. Partial Differential Equations 32: 1281-1343, 2007). Moreover, we investigate the dynamical properties of blow-up solutions, including $L^{2}$-concentration, blow-up rates and limiting profile. When $\frac{4}{N}<p_{1}<\frac{4}{N-2}\left(4<p_{1}<\infty\right.$ if $N=1,2<p_{1}<\infty$ if $N=2$ ), we prove that the blow-up solution with bounded $\dot{H}^{s_{c}}$-norm must concentrate at least a fixed amount of the $\dot{H}^{s_{c}}$-norm and, also, its $L^{p_{c}}$-norm must concentrate at least a fixed $L^{p_{c}}$-norm.
\end{abstract}

Keywords: Nonlinear Schrödinger equation; Blow-up solutions; Concentration; Limiting profile

\section{Introduction}

Because of important applications in physics, nonlinear Schrödinger equations attracted a great deal of attention from mathematicians in the past decades, see [1, 18, 19] for a review. We recall some known results about blow-up solutions for the classical nonlinear Schrödinger equation

$$
i u_{t}+\Delta u=\lambda|u|^{p} u
$$

E-mail: binhuaf@163.com(Binhua Feng)

This work is supported by NSFC Grants (No. 11601435, No. 11401478), Gansu Provincial Natural Science Foundation (1606RJZA010) and NWNU-LKQN-14-6. 
Ginibre and Velo [7] established the local well-posedness of (1.1) in $H^{1}$ ( see [1] for a review). Glassey [8] proved the existence of blow-up solutions for the negative energy and $|x| u_{0} \in L^{2}$. Ogawa and Tsutsumi [17] proved the existence of blow-up solutions in radial case without the restriction $|x| u_{0} \in L^{2}$. Weinstein [21], Zhang [23] obtained the sharp conditions of global existence for $L^{2}$-critical and $L^{2}$-supercritical nonlinearities. Moreover, for the $L^{2}$-critical nonlinearity, Weinstein [22] studied the structure and formation of singularity of blow-up solutions with critical mass by the concentration compact principle: the blow-up solution is close to the ground state in $H^{1}$ up to scaling and phase parameters, and also translation in the non-radial case. Applying the variational methods, Merle and Raphaël [13] improved Weinstein's results and obtained the sharp decomposition of blow-up solutions with small super-critical mass. By this sharp decomposition and spectral properties, Merle and Raphaël [12, 13, 14, 15] obtained a large body of breakthrough works, such as sharp blow-up rates, profiles, etc. Hmidi and Keraani [10] established the profile decomposition of bounded sequences in $H^{1}$ and gave a new and simple proof for some dynamical properties of blow-up solutions in $H^{1}$. These results have been generalized to other kinds of Schrödinger equations, see [3, 4, 5, 9, 11, 24, 25, 26, 27].

In this paper, we will investigate blow-up solutions of the nonlinear Schrödinger equation with combined power-type nonlinearities

$$
\left\{\begin{array}{l}
i u_{t}+\Delta u=\lambda_{1}|u|^{p_{1}} u+\lambda_{2}|u|^{p_{2}} u, \\
u(0, x)=u_{0}(x),
\end{array}\right.
$$

where $u(t, x):\left[0, T^{*}\right) \times \mathbb{R}^{N} \rightarrow \mathbb{C}$ is a complex valued function and $0<T^{*} \leq \infty, 0<p_{2}<p_{1}<$ $\frac{4}{N-2}\left(0<p_{2}<p_{1} \leq \infty\right.$ if $N=1,0<p_{2}<p_{1}<\infty$ if $\left.N=2\right)$. This equation arises as the leadingorder model for propagation of intense laser beams in an isotropic bulk medium, see Section 32.1 in [6] for a detailed explanation. Equation (1.2) can also be considered as a simplified model resulting from the expansion of the nonlinear Schrödinger equation with saturated nonlinearity, which is relevant in the description of Bose superfluids at zero temperature, in the Hartree approximation, see [18].

In [20], Tao et al. undertook a comprehensive study for (1.2). More precisely, they addressed questions related to local and global well-posedness, finite time blow-up, and asymptotic 
behaviour. This equation has Hamiltonian

$$
E(u(t)):=\frac{1}{2} \int_{\mathbb{R}^{N}}|\nabla u(t, x)|^{2} d x+\frac{\lambda_{1}}{p_{1}+2} \int_{\mathbb{R}^{N}}|u(t, x)|^{p_{1}+2} d x+\frac{\lambda_{2}}{p_{2}+2} \int_{\mathbb{R}^{N}}|u(t, x)|^{p_{2}+2} d x .
$$

But there is no scaling invariance for this equation when $p_{1} \neq p_{2}$.

About the existence of blow-up solutions, they proved the following theorem.

Theorem A. Let $u_{0} \in \Sigma:=\left\{u \in H^{1}, x u \in L^{2}\right\}, \lambda_{1}<0$, and $\frac{4}{N}<p_{1} \leq \frac{4}{N-2}$ with $N \geq 3$. Let $y_{0}:=\operatorname{Im} \int r \bar{u}_{0} \partial_{r} u_{0} d x$ denote the weighted mass current and assume $y_{0}>0$. Then, blow-up occurs in each of the following three cases:

1) $\lambda_{2}>0,0<p_{2}<p_{1}$, and $E\left(u_{0}\right)<0$;

2) $\lambda_{2}<0, \frac{4}{N}<p_{2}<p_{1}$, and $E\left(u_{0}\right)<0$;

3) $\lambda_{2}<0,0<p_{2} \leq \frac{4}{N}$, and $E\left(u_{0}\right)+C M\left(u_{0}\right)<0$ for some suitably large constant C.

More precisely, in any of the above cases there exists $0<T^{*} \leq C \frac{\left\|x u_{0}\right\|_{L^{2}}^{2}}{y_{0}}$ such that

$$
\lim _{t \rightarrow T^{*}}\|\nabla u(t)\|_{L^{2}}=\infty
$$

As far as we know, when $\lambda_{1}<0, \lambda_{2}>0, p_{1}=\frac{4}{N}, 0<p_{2}<\frac{4}{N}$, the existence of blowup solutions of (1.2) has not been proved yet. In this paper, we first prove the existence of blow-up solutions, and then give the sharp threshold mass of blow-up and global existence in this case. Moreover, we will investigate some dynamical properties of blow-up solutions of (1.2) with $L^{2}$-critical or $L^{2}$-supercritical nonlinearity, including $L^{2}$-concentration, $\dot{H}^{s_{c}}$-concentration,

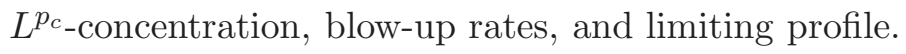

To solve these problems, we mainly use the ideas from Hmidi and Keraani [10] and Guo 9]. The dynamics of blow-up solutions for the $L^{2}$-critical and $L^{2}$-supercritical nonlinear Schrödinger equation (1.1) has been discussed in Hmidi and Keraani [10] and Guo [9], respectively. In these papers, the study of dynamics of blow-up solutions relies heavily on the scale invariance of (1.1). Hence, the study of dynamics of blow-up solutions for (1.2), which has no the scale invariance, is of particular interest. First, we prove the existence of blow-up solutions and find the sharp threshold mass $\|Q\|_{L^{2}}$ of blow-up and global existence for (1.2), where $Q$ is the ground state solution of (2.3). Then, in order to overcome the loss of scale invariance, we use the ground state solution $Q$ of (2.3) to describe the dynamical behavior of blow-up solutions to (1.2). In 
the $L^{2}$-supercritical case, we choose the ground states of equations (5.1) and (5.2) to describe some concentration properties of blow-up solutions to (1.2).

This paper is organized as follows: in Section 2, we present some preliminaries. In section 3, we first prove the existence of blow-up solutions of (1.2), and then give the sharp threshold mass of blow-up and global existence. In section 4, we will consider some dynamical properties of blow-up solutions of (1.2) with $p_{1}=\frac{4}{N}$ and $0<p_{2}<\frac{4}{N}$, including $L^{2}$-concentration, blow-up rate, and limiting profile. In section 5 , we will obtain some concentration properties of blow-up solutions of (1.2) with $\frac{4}{N}<p_{1}<\frac{4}{N-2}$ and $0<p_{2}<p_{1}$.

Notation. Throughout this paper, we use the following notation. $C>0$ will stand for a constant that may be different from line to line when it does not cause any confusion. We often use the abbreviations $L^{r}=L^{r}\left(\mathbb{R}^{N}\right), H^{s}=H^{s}\left(\mathbb{R}^{N}\right)$ in what follows. $\Sigma:=\left\{u \in H^{1}, x u \in L^{2}\right\}$ denotes the energy space equipped with the norm $\|u\|_{\Sigma}:=\|u\|_{H^{1}}+\|x u\|_{L^{2}}$. For $s \in \mathbb{R}$, the pseudo-

differential operator $(-\Delta)^{s}$ is defined by $\widehat{(-\Delta)^{s}} f(\xi)=|\xi|^{2 s} \hat{f}(\xi)$, where^ denotes Fourier transform. We also use homogeneous Sobolev space $\dot{H}^{s}\left(\mathbb{R}^{N}\right)=\left\{u \in \mathcal{S}^{\prime}\left(\mathbb{R}^{N}\right) ; \int|\xi|^{2 s}|\hat{f}(\xi)|^{2} d \xi<\infty\right\}$ with its norm defined by $\|f\|_{\dot{H}^{s}}=\left\|(-\Delta)^{s / 2} f\right\|_{L^{2}}$, where $\mathcal{S}^{\prime}\left(\mathbb{R}^{N}\right)$ denotes the space of tempered distribution on $\mathbb{R}^{N}$. In particular, we use notation: $s_{c}=\frac{N}{2}-\frac{2}{p_{1}}$ and $p_{c}=\frac{N p_{1}}{2}$. Therefore, it follows from the Sobolev embedding that $\dot{H}^{s_{c}}\left(\mathbb{R}^{N}\right) \hookrightarrow L^{p_{c}}\left(\mathbb{R}^{N}\right)$.

\section{Preliminaries}

Firstly, let us recall the local theory for the initial value problem (1.2) established in [1].

Proposition 2.1. Let $u_{0} \in H^{1}, 0<p_{1}, p_{2}<\frac{4}{N-2}\left(0<p_{1}, p_{2}<\infty\right.$ if $N=1,0<p_{1}, p_{2}<\infty$ if $N=2)$. Then, there exists $T=T\left(\left\|u_{0}\right\|_{H^{1}}\right)$ such that (1.2) admits a unique solution $u \in$ $C\left([0, T], H^{1}\right)$. Let $\left[0, T^{*}\right)$ be the maximal time interval on which the solution $u$ is well-defined, if $T^{*}<\infty$, then $\|u(t)\|_{H^{1}} \rightarrow \infty$ as $t \uparrow T^{*}$. Moreover, for all $0 \leq t<T^{*}$, the solution $u(t)$ satisfies the following conservation of mass and energy

$$
\begin{gathered}
\|u(t)\|_{L^{2}}=\left\|u_{0}\right\|_{L^{2}}, \\
E(u(t))=E\left(u_{0}\right),
\end{gathered}
$$


where $E(u(t))$ defined by (1.3).

For more specific results concerning the Cauchy problem (1.2), we refer the reader to [1]. In addition, by some basic calculations, we have the following result(see [1]).

Lemma 2.2. Assume that $u_{0} \in \Sigma$, and the corresponding solution $u$ of (1.2) exists on the interval $\left[0, T^{*}\right)$. Then, for all $t \in\left[0, T^{*}\right)$, it follows $u(t) \in \Sigma$. Moreover, let $J(t)=$ $\int_{\mathbb{R}^{N}}|x u(t, x)|^{2} d x$, then

$$
J^{\prime}(t)=-4 \operatorname{Im} \int_{\mathbb{R}^{N}} u(t, x) x \cdot \nabla \bar{u}(t, x) d x
$$

and

$$
J^{\prime \prime}(t)=8 \int_{\mathbb{R}^{N}}|\nabla u(t, x)|^{2} d x+\frac{4 N \lambda_{1} p_{1}}{p_{1}+2} \int_{\mathbb{R}^{N}}|u(t, x)|^{p_{1}+2} d x+\frac{4 N \lambda_{2} p_{2}}{p_{2}+2} \int_{\mathbb{R}^{N}}|u(t, x)|^{p_{2}+2} d x
$$

Finally, we recall the following useful result of M. Weinstein [21] relating the ground states of (2.3) with the best constant in a Gagliardo-Nirenberg inequality.

Lemma 2.3. [21] Let $p=\frac{4}{N}$ and $Q$ be the ground state solution of the following elliptic equation

$$
-\Delta Q+Q=|Q|^{p+2} Q \quad \text { in } \mathbb{R}^{N}
$$

It follows that the optimal constant in the Gagliardo-Nirenberg inequality

$$
\frac{1}{p+2}\|u\|_{L^{p+2}}^{p+2} \leq \frac{C}{2}\|u\|_{L^{2}}^{p}\|\nabla u\|_{L^{2}}^{2}
$$

is $C=\|Q\|_{L^{2}}^{-p}$.

Remark. When $N=1$, this lemma was proved by Nagy in [16].

\section{The sharp threshold mass of blow-up and global existence}

By the local well-posedness theory of the nonlinear Schrödinger equation, the solution of (1.2) with small initial data exists globally, and for some large initial data, the solution may blow up in finite time. Thus, whether there exists a sharp threshold of blow-up and global existence for (1.2) is of particular interest. On the other hand, the following problems are very important 
from the view-point of physics. Under what conditions will the condensate become unstable to collapse (blow-up)? And under what conditions will the condensate exist for all time (global existence)? Especially the sharp thresholds for blow-up and global existence are pursued strongly (see [1, 2, 18, 21, 23, 24] and their references).

To solve this problem for (1.2), there exists two major difficulties. One is the loss of scale invariance for (1.2); the other is that the second order derivative of $J(t)=\int_{\mathbb{R}^{N}}|x u(t, x)|^{2} d x$ is the following form:

$$
J^{\prime \prime}(t)=16 E\left(u_{0}\right)+\frac{4 N p_{2}-16}{p_{2}+2} \int_{\mathbb{R}^{N}}|u(t, x)|^{p_{2}+2} d x .
$$

Because $\int_{\mathbb{R}^{N}}|u(t, x)|^{p_{2}+2} d x$ is a positive uncertain function, which may be unbounded with respect to time $t$, it is hard to choose $E\left(u_{0}\right)$ to ensure the existence of blow-up solutions.

In the following theorem, by using the scaling argument and the inequality (2.4), we obtain the existence of blow-up solutions for (1.2) and the sharp threshold mass of blow-up and global existence for (1.2).

Theorem 3.1. Let $u_{0} \in H^{1}, \lambda_{1}=-1, \lambda_{2}=1, p_{1}=\frac{4}{N}$ and $0<p_{2}<\frac{4}{N}$. Assume that $Q$ is the ground state solution of (2.3). Then, we have the following sharp threshold mass of blow-up and global existence.

(i) If $\left\|u_{0}\right\|_{L^{2}}<\|Q\|_{L^{2}}$, then the solution of (1.2) exists globally.

(ii) If the initial data $u_{0}=c \rho^{\frac{N}{2}} Q(\rho x)$ satisfies $|x| u_{0} \in L^{2}$, where the complex number $c$ satisfying $|c| \geq 1$, and the real number $\rho>0$, then the solution $u$ of (1.2) with initial data $u_{0}$ blows up in finite time.

Remark: From Remark 6.6.2 in [1], we infer that the critical value about the initial data for global existence of (1.1) and (1.2) is the same.

Proof. (i) We deduce from (1.3) and (2.4) that

$$
\begin{aligned}
E\left(u_{0}\right)=E(u(t)) & =\frac{1}{2} \int_{\mathbb{R}^{N}}|\nabla u(t, x)|^{2} d x-\frac{1}{p_{1}+2} \int_{\mathbb{R}^{N}}|u(t, x)|^{p_{1}+2} d x+\frac{1}{p_{2}+2} \int_{\mathbb{R}^{N}}|u(t, x)|^{p_{2}+2} d x \\
& \geq\left(\frac{1}{2}-\frac{\left\|u_{0}\right\|_{L^{2}}^{p_{1}}}{2\|Q\|_{L^{2}}^{p_{1}}}\right)\|\nabla u(t)\|_{L^{2}}^{2} .
\end{aligned}
$$


Due to $\left\|u_{0}\right\|_{L^{2}}<\|Q\|_{L^{2}}$, we have that $\|\nabla u(t)\|_{L^{2}}$ is uniformly bounded for all time $t$. Therefore, (i) follows from the conservation of mass and Proposition 2.1.

(ii) Since $|x| u_{0} \in L^{2}, J(t)=\int_{\mathbb{R}^{N}}|x u(t, x)|^{2} d x$ is well-defined, and it follows from Lemma 2.2 that

$$
J^{\prime \prime}(t)=16 E\left(u_{0}\right)+\frac{4 N p_{2}-16}{p_{2}+2} \int_{\mathbb{R}^{N}}|u(t, x)|^{p_{2}+2} d x .
$$

By the definition of initial data $u_{0}(x)=c \rho^{\frac{N}{2}} Q(\rho x)$ and the Pohožaev identity for equation (2.3), i.e., $\frac{1}{2}\|\nabla Q\|_{L^{2}}^{2}=\frac{1}{p_{1}+2}\|Q\|_{L^{p_{1}+2}}^{p_{1}}$, we deduce that

$$
\begin{aligned}
E\left(u_{0}\right) & =\frac{|c|^{2} \rho^{2}}{2} \int_{\mathbb{R}^{N}}|\nabla Q(x)|^{2} d x-\frac{|c|^{p_{1}+2} \rho^{2}}{p_{1}+2} \int_{\mathbb{R}^{N}}|Q(x)|^{p_{1}+2} d x+\frac{|c|^{p_{2}+2} \rho^{\frac{N}{2} p_{2}}}{p_{2}+2} \int_{\mathbb{R}^{N}}|Q(x)|^{p_{2}+2} d x \\
& =-\frac{|c|^{2} \rho^{2}}{2}\left(|c|^{p_{1}}-1\right)\|\nabla Q\|_{L^{2}}^{2}+\frac{|c|^{p_{2}+2} \rho^{\frac{N}{2} p_{2}}}{p_{2}+2} \int_{\mathbb{R}^{N}}|Q(x)|^{p_{2}+2} d x .
\end{aligned}
$$

Now, taking $\rho$ such that

$$
\frac{2|c|^{p_{2}}\|Q\|_{L^{p_{2}+2}}^{p_{2}+2}}{\left(p_{2}+2\right)\left(|c|^{p_{1}}-1\right)\|\nabla Q\|_{L^{2}}^{2}}<\rho^{2-\frac{N}{2} p_{2}} .
$$

This implies $E\left(u_{0}\right)<0$. It follows from (3.1) that $J^{\prime \prime}(t)<16 E\left(u_{0}\right)<0$. By the standard concave argument, the solution $u$ of (1.2) with the initial data $u_{0}$ blows up in finite time.

\section{Dynamic of blow-up solutions in the case of $L^{2}$-critical}

In this section, we investigate some dynamical properties of blow-up solutions for (1.2) with $\lambda_{1}=-1, \lambda_{2}=1, p_{1}=\frac{4}{N}$, and $0<p_{2}<\frac{4}{N}$. These results are closed related to the results obtained for the classical nonlinear Schrödinger equation (1.1) with $p=\frac{4}{N}$ by Hmidi and Keraani [10]. For this aim, we firstly recall the following refined compactness result which can be proved by using the profile decomposition of bounded sequences in $H^{1}$ and the inequality (2.4), see [10].

Lemma 4.1. Let $\left\{u_{n}\right\}_{n=1}^{\infty}$ be a bounded sequence in $H^{1}$, such that

$$
\limsup _{n \rightarrow \infty}\left\|\nabla u_{n}\right\|_{L^{2}} \leq M, \quad \limsup _{n \rightarrow \infty}\left\|u_{n}\right\|_{L^{4 / N+2}} \geq m>0 .
$$

Then, there exist $V \in H^{1}$ and $\left\{x_{n}\right\}_{n=1}^{\infty} \subset \mathbb{R}^{N}$ such that, up to a subsequence,

$$
u_{n}\left(\cdot+x_{n}\right) \rightarrow V \text { weakly in } H^{1}
$$


with

$$
\|V\|_{L^{2}} \geq\left(\frac{N}{N+2}\right)^{\frac{N}{4}} \frac{m^{N / 2+1}}{M^{N / 2}}\|Q\|_{L^{2}} .
$$

where $Q$ is the ground state solution of (2.3).

Theorem 4.2. (L $L^{2}$-concentration) Let $u_{0} \in H^{1}, \lambda_{1}=-1, \lambda_{2}=1, p_{1}=\frac{4}{N}$, and $0<p_{2}<\frac{4}{N}$. If the solution $u$ of (1.2) blows up in finite time $T^{*}>0$. Let a $(t)$ be a real-valued nonnegative function defined on $\left[0, T^{*}\right)$ satisfying $a(t)\|\nabla u(t)\|_{L^{2}} \rightarrow \infty$ as $t \rightarrow T^{*}$. Then there exists $x(t) \in$ $\mathbb{R}^{N}$ such that

$$
\liminf _{t \rightarrow T^{*}} \int_{|x-x(t)| \leq a(t)}|u(t, x)|^{2} d x \geq \int_{\mathbb{R}^{N}}|Q(x)|^{2} d x .
$$

where $Q$ is the ground state solution of (2.3).

Remark. Theorem 4.2 gives the $L^{2}$-concentration and rate of $L^{2}$-concentration of blow-up solutions of (1.2). Indeed, we can choose $a(t)=\frac{1}{\|\nabla u(t)\|_{L^{2}}^{1-\delta}}$ with $0<\delta<1$. It is obvious that $\lim _{t \rightarrow T^{*}} a(t)=0$ and $a(t)$ satisfies the assumption in Theorem 4.2. Applying Theorem 4.2, if $u$ is a blow-up solution of (1.2) and $T^{*}$ its blow-up time, then for every $r>0$, there exists a function $x(t) \in \mathbb{R}^{N}$ such that

$$
\liminf _{t \rightarrow T^{*}} \int_{|x-x(t)| \leq r}|u(t, x)|^{2} d x \geq \int_{\mathbb{R}^{N}}|Q(x)|^{2} d x .
$$

Meanwhile, it follows from the choice of $a(t)$ that for any function $a(t) \leq \frac{1}{\|\nabla u(t)\|_{L^{2}}^{1-\delta}}$, (4.1) holds, which implies that the rate of $L^{2}$-concentration of blow-up solutions of $(1.2)$ is $\frac{1}{\|\nabla u(t)\|_{L^{2}}^{1-\delta}}$ with $0<\delta<1$.

Proof. Set

$$
\rho(t)=\|\nabla Q\|_{L^{2}} /\|\nabla u(t)\|_{L^{2}} \text { and } v(t, x)=\rho^{\frac{N}{2}}(t) u(t, \rho(t) x) .
$$

Let $\left\{t_{n}\right\}_{n=1}^{\infty}$ be an any time sequence such that $t_{n} \rightarrow T^{*}, \rho_{n}:=\rho\left(t_{n}\right)$ and $v_{n}(x):=v\left(t_{n}, x\right)$. Then, the sequence $\left\{v_{n}\right\}$ satisfies

$$
\left\|v_{n}\right\|_{L^{2}}=\left\|u\left(t_{n}\right)\right\|_{L^{2}}=\left\|u_{0}\right\|_{L^{2}}, \quad\left\|\nabla v_{n}\right\|_{L^{2}}=\rho_{n}\left\|\nabla u\left(t_{n}\right)\right\|_{L^{2}}=\|\nabla Q\|_{L^{2}} .
$$


Observe that

$$
\begin{aligned}
H\left(v_{n}\right): & =\frac{1}{2} \int_{\mathbb{R}^{N}}\left|\nabla v_{n}(x)\right|^{2} d x-\frac{1}{p_{1}+2} \int_{\mathbb{R}^{N}}\left|v_{n}(x)\right|^{p_{1}+2} d x \\
& =\rho_{n}^{2}\left(\frac{1}{2} \int_{\mathbb{R}^{N}}\left|\nabla u\left(t_{n}, x\right)\right|^{2} d x-\frac{1}{p_{1}+2} \int_{\mathbb{R}^{N}}\left|u\left(t_{n}, x\right)\right|^{p_{1}+2} d x\right) \\
& =\rho_{n}^{2}\left(E\left(u_{0}\right)-\frac{1}{p_{2}+2} \int_{\mathbb{R}^{N}}\left|u\left(t_{n}, x\right)\right|^{p_{2}+2} d x\right) .
\end{aligned}
$$

Thus, applying the following Gagliardo-Nirenberg inequality

$$
\int_{\mathbb{R}^{N}}|u(x)|^{p_{2}+2} d x \leq C\|u\|_{L^{2}}^{p_{2}+2-\frac{N p_{2}}{2}}\|\nabla u\|_{L^{2}}^{\frac{N p_{2}}{2}}, \text { for } 0<p_{2}<\frac{4}{N}
$$

we deduce that

$$
\begin{aligned}
\left|H\left(v_{n}\right)\right| & \leq \rho_{n}^{2}\left(\left|E\left(u_{0}\right)\right|+\frac{1}{p_{2}+2} \int_{\mathbb{R}^{N}}\left|u\left(t_{n}, x\right)\right|^{p_{2}+2} d x\right) \\
& \leq \frac{\left|E\left(u_{0}\right)\right|\|\nabla Q\|_{L^{2}}^{2}}{\left\|\nabla u\left(t_{n}\right)\right\|_{L^{2}}^{2}}+C \frac{\|\nabla Q\|_{L^{2}}^{2}\left\|\nabla u\left(t_{n}\right)\right\|_{L^{2}}^{\frac{N p_{2}}{2}}}{\left\|\nabla u\left(t_{n}\right)\right\|_{L^{2}}^{2}} \\
& \rightarrow 0 \text { as } n \rightarrow \infty,
\end{aligned}
$$

which implies $\int_{\mathbb{R}^{N}}\left|v_{n}(x)\right|^{p_{1}+2} d x \rightarrow(2 / N+1)\|\nabla Q\|_{L^{2}}^{2}$.

Set $m^{p_{1}+2}=(2 / N+1)\|\nabla Q\|_{L^{2}}^{2}$ and $M=\|\nabla Q\|_{L^{2}}$. Then it follows from Lemma 4.1 that there exist $V \in H^{1}$ and $\left\{x_{n}\right\}_{n=1}^{\infty} \subset \mathbb{R}^{N}$ such that, up to a subsequence,

$$
v_{n}\left(\cdot+x_{n}\right)=\rho_{n}^{N / 2} u\left(t_{n}, \rho_{n}\left(\cdot+x_{n}\right)\right) \rightarrow V \text { weakly in } H^{1}
$$

with

$$
\|V\|_{L^{2}} \geq\|Q\|_{L^{2}}
$$

Note that

$$
\frac{a\left(t_{n}\right)}{\rho_{n}}=\frac{a\left(t_{n}\right)\left\|\nabla u\left(t_{n}\right)\right\|_{L^{2}}}{\|\nabla Q\|_{L^{2}}} \rightarrow \infty, \text { as } n \rightarrow \infty .
$$

Then for every $r>0$, there exists $n_{0}>0$ such that for every $n>n_{0}, r \rho_{n}<a\left(t_{n}\right)$. Therefore, 
using (4.5), we obtain

$$
\begin{aligned}
\liminf _{n \rightarrow \infty} \sup _{y \in \mathbb{R}^{N}} \int_{|x-y| \leq a\left(t_{n}\right)}\left|u\left(t_{n}, x\right)\right|^{2} d x & \geq \liminf _{n \rightarrow \infty} \sup _{y \in \mathbb{R}^{N}} \int_{|x-y| \leq r \rho_{n}}\left|u\left(t_{n}, x\right)\right|^{2} d x \\
& \geq \liminf _{n \rightarrow \infty} \int_{\left|x-x_{n}\right| \leq r \rho_{n}}\left|u\left(t_{n}, x\right)\right|^{2} d x \\
& =\liminf _{n \rightarrow \infty} \int_{|x| \leq r} \rho_{n}^{N}\left|u\left(t_{n}, \rho_{n}\left(x+x_{n}\right)\right)\right|^{2} d x \\
& =\liminf _{n \rightarrow \infty} \int_{|x| \leq r}\left|v\left(t_{n}, x+x_{n}\right)\right|^{2} d x \\
& \geq \liminf _{n \rightarrow \infty} \int_{|x| \leq r}|V(x)|^{2} d x, \quad \text { for every } r>0,
\end{aligned}
$$

which means that

$$
\liminf _{n \rightarrow \infty} \sup _{y \in \mathbb{R}^{N}} \int_{|x-y| \leq a\left(t_{n}\right)}\left|u\left(t_{n}, x\right)\right|^{2} d x \geq \int_{\mathbb{R}^{N}}|V(x)|^{2} d x
$$

Since the sequence $\left\{t_{n}\right\}_{n=1}^{\infty}$ is arbitrary, we obtain

$$
\liminf _{t \rightarrow T^{*}} \sup _{y \in \mathbb{R}^{N}} \int_{|x-y| \leq a(t)}|u(t, x)|^{2} d x \geq \int_{\mathbb{R}^{N}}|Q(x)|^{2} d x
$$

Observe that for every $t \in\left[0, T^{*}\right)$, the function $g(y):=\int_{|x-y| \leq a(t)}|u(t, x)|^{2} d x$ is continuous on $y \in \mathbb{R}^{N}$ and $g(y) \rightarrow 0$ as $|y| \rightarrow \infty$. So there exists a function $x(t) \in \mathbb{R}^{N}$ such that for every $t \in\left[0, T^{*}\right)$

$$
\sup _{y \in \mathbb{R}^{N}} \int_{|x-y| \leq a(t)}|u(t, x)|^{2} d x=\int_{|x-x(t)| \leq a(t)}|u(t, x)|^{2} d x .
$$

This and (4.7) yield (4.1).

In the following theorem, we study the limiting profile of blow-up solutions of (1.2).

Theorem 4.3. Let $u_{0} \in H^{1}, \lambda_{1}=-1, \lambda_{2}=1, p_{1}=\frac{4}{N}$, and $0<p_{2}<\frac{4}{N}$. Assume $\left\|u_{0}\right\|_{L^{2}}=\|Q\|_{L^{2}}$, and the corresponding solution $u$ of (1.2) blows up in finite time $T^{*}>0$, then there exist $x(t) \in \mathbb{R}^{N}$ and $\theta(t) \in[0,2 \pi)$ such that

$$
\rho^{N / 2}(t) u(t, \rho(t)(\cdot+x(t))) e^{i \theta(t)} \rightarrow Q \text { strongly in } H^{1} \text {, as } t \rightarrow T^{*},
$$

where $\rho(t)=\frac{\|\nabla Q\|_{L^{2}}}{\|\nabla u(t)\|_{L^{2}}}$. 
Proof. We use the notations in the proof of Theorem 4.2. Assume that $\left\|u_{0}\right\|_{L^{2}}=\|Q\|_{L^{2}}$. Recall that we have verified that $\|V\|_{L^{2}} \geq\|Q\|_{L^{2}}$ in the proof of Theorem 4.2. Whence

$$
\|Q\|_{L^{2}} \leq\|V\|_{L^{2}} \leq \liminf _{n \rightarrow \infty}\left\|v_{n}\right\|_{L^{2}}=\liminf _{n \rightarrow \infty}\left\|u\left(t_{n}\right)\right\|_{L^{2}}=\left\|u_{0}\right\|_{L^{2}}=\|Q\|_{L^{2}},
$$

and then,

$$
\lim _{n \rightarrow \infty}\left\|v_{n}\right\|_{L^{2}}=\|V\|_{L^{2}}=\|Q\|_{L^{2}},
$$

which implies

$$
v_{n}\left(\cdot+x_{n}\right) \rightarrow V \text { strongly in } L^{2} \text { as } n \rightarrow \infty \text {. }
$$

We infer from the inequality (2.4) that

$$
\left\|v_{n}\left(\cdot+x_{n}\right)-V\right\|_{L^{p_{1}+2}}^{p_{1} 2} \leq C\left\|v_{n}\left(\cdot+x_{n}\right)-V\right\|_{L^{2}}^{p_{1}}\left\|\nabla\left(v_{n}\left(\cdot+x_{n}\right)-V\right)\right\|_{L^{2}}^{2} .
$$

From $\left\|\nabla v_{n}\left(\cdot+x_{n}\right)\right\|_{L^{2}} \leq C$, we get

$$
v_{n}\left(\cdot+x_{n}\right) \rightarrow V \text { in } L^{p_{1}+2} \text { as } n \rightarrow \infty
$$

Next, we will prove that $v_{n}\left(\cdot+x_{n}\right)$ converges to $V$ strongly in $H^{1}$. For this aim, we estimate as follows:

$$
\begin{aligned}
0 & =\lim _{n \rightarrow \infty} H\left(v_{n}\right) \\
& =\frac{1}{2} \int_{\mathbb{R}^{N}}|\nabla Q(x)|^{2} d x-\frac{1}{p_{1}+2} \lim _{n \rightarrow \infty} \int_{\mathbb{R}^{N}}\left|v_{n}(x)\right|^{p_{1}+2} d x \\
& =\frac{1}{2} \int_{\mathbb{R}^{N}}|\nabla Q(x)|^{2} d x-\frac{1}{p_{1}+2} \int_{\mathbb{R}^{N}}|V(x)|^{p_{1}+2} d x .
\end{aligned}
$$

Thus, we infer from the inequality (2.4) that

$$
\frac{1}{2} \int_{\mathbb{R}^{N}}|\nabla Q(x)|^{2} d x=\frac{1}{p_{1}+2} \int_{\mathbb{R}^{N}}|V(x)|^{p_{1}+2} d x \leq \frac{1}{2} \frac{\|V\|_{L^{2}}^{p_{1}}}{\|Q\|_{L^{2}}^{p_{1}}}\|\nabla V\|_{L^{2}}^{2}=\frac{1}{2}\|\nabla V\|_{L^{2}}^{2} .
$$

On the other hand, we deduce from (4.2) that $\|\nabla V\|_{L^{2}} \leq \liminf _{n \rightarrow \infty}\left\|\nabla v_{n}\left(\cdot+x_{n}\right)\right\|_{L^{2}}=\|\nabla Q\|_{L^{2}}$. Hence, we have $\|Q\|_{H^{1}}=\|V\|_{H^{1}}$ and

$$
v_{n}\left(\cdot+x_{n}\right) \rightarrow V \text { strongly in } H^{1} \text { as } n \rightarrow \infty \text {. }
$$

This and (4.11) imply that

$$
H(V)=\frac{1}{2} \int_{\mathbb{R}^{N}}|\nabla V(x)|^{2} d x-\frac{1}{p_{1}+2} \int_{\mathbb{R}^{N}}|V(x)|^{p_{1}+2} d x=0 .
$$


Up to now, we have verified that

$$
\|V\|_{L^{2}}=\|Q\|_{L^{2}},\|\nabla V\|_{L^{2}}=\|\nabla Q\|_{L^{2}} \text { and } H(V)=0 .
$$

The variational characterization of the ground state implies that

$$
V(x)=e^{i \theta} Q\left(x+x_{0}\right) \text { for some } \theta \in[0,2 \pi), x_{0} \in \mathbb{R}^{N}
$$

and

$$
\rho_{n}^{N / 2} u\left(t_{n}, \rho_{n}\left(\cdot+x_{0}\right)\right) \rightarrow e^{i \theta} Q\left(\cdot+x_{0}\right) \text { strongly in } H^{1} \text { as } n \rightarrow \infty \text {. }
$$

Since the sequence $\left\{t_{n}\right\}_{n=1}^{\infty}$ is arbitrary, we infer that there are two functions $x(t) \in \mathbb{R}^{N}$ and $\theta(t) \in[0,2 \pi)$ such that

$$
\rho^{N / 2}(t) e^{i \theta(t)} u(t, \rho(t)(x+x(t))) \rightarrow Q \text { strongly in } H^{1} \text { as } t \rightarrow T^{*} .
$$

In the following theorem, we will prove that the blow-up solution $|u(t, x)|^{2}$ like a $\delta$-function as $t \rightarrow T^{*}$ at the point $x=x_{0}$, which implies that the point $x_{0}$ concentrates all mass of blow-up solution of (1.2).

Theorem 4.4. Let $u_{0} \in \Sigma, \lambda_{1}=-1, \lambda_{2}=1, p_{1}=\frac{4}{N}$, and $0<p_{2}<\frac{4}{N}$. If the solution $u$ of (1.2) blows up in finite time $T^{*}>0$ and $\left\|u_{0}\right\|_{L^{2}}=\|Q\|_{L^{2}}$, then there exists $x_{0} \in \mathbb{R}^{N}$ such that

$$
|u(t, x)|^{2} \rightarrow\|Q\|_{L^{2}}^{2} \delta_{x_{0}}
$$

in the sense of distribution as $t \rightarrow T^{*}$.

Proof. According to Theorem 4.2, it follows that for all $r>0$

$$
\liminf _{t \rightarrow T^{*}} \int_{|x-x(t)|<r}|u(t, x)|^{2} d x \geq\|Q\|_{L^{2}}^{2}
$$

This, together with the conservation of mass $\|u(t)\|_{L^{2}}^{2}=\left\|u_{0}\right\|_{L^{2}}^{2}=\|Q\|_{L^{2}}^{2}$, implies that for all $r>0$

$$
\liminf _{t \rightarrow T^{*}} \int_{|x-x(t)|<r}|u(t, x)|^{2} d x=\|Q\|_{L^{2}}^{2}
$$


This yields

$$
|u(t, x+x(t))|^{2} \rightarrow\|Q\|_{L^{2}}^{2} \delta_{x=0}
$$

By using the inequality (2.4), for any $\varepsilon>0$ and any real-valued function $\theta$ defined on $\mathbb{R}^{N}$, we have

$$
H\left(e^{ \pm i \epsilon \theta} u\right) \geq \frac{1}{2} \int_{\mathbb{R}^{N}}\left|\nabla\left(e^{ \pm i \epsilon \theta} u\right)\right|^{2} d x\left(1-\frac{\|u\|_{L^{2}}^{p_{1}}}{\|Q\|_{L^{2}}^{p_{1}}}\right)=0
$$

Therefore,

$$
0 \leq H\left(e^{ \pm i \epsilon \theta} u\right)=\frac{\epsilon^{2}}{2} \int_{\mathbb{R}^{N}}|u|^{2}|\nabla \theta|^{2} d x \mp \epsilon \operatorname{Im} \int_{\mathbb{R}^{N}} \bar{u} \nabla u \cdot \nabla \theta d x+H(u)
$$

which implies that

$$
\left|\mp I m \int_{\mathbb{R}^{N}} \bar{u} \nabla u \cdot \nabla \theta d x\right| \leq\left(2 H(u) \int_{\mathbb{R}^{N}}|u|^{2}|\nabla \theta|^{2} d x\right)^{1 / 2} .
$$

For any $j=1,2, \ldots, N$, it follows from (4.16) and $H(u(t)) \leq E(u(t))=E\left(u_{0}\right)$ that

$$
\begin{aligned}
\left.\left|\frac{d}{d t} \int_{\mathbb{R}^{N}}\right| u(t, x)\right|^{2} x_{j} d x \mid & =2\left|\int_{\mathbb{R}^{N}} \bar{u} \partial_{j} u d x\right| \\
& =2\left|\int_{\mathbb{R}^{N}} \bar{u} \nabla u \nabla x_{j} d x\right| \\
& \leq 2\left(2 H(u) \int_{\mathbb{R}^{N}}|u|^{2}\left|\nabla x_{j}\right|^{2} d x\right)^{1 / 2} \\
& \leq C .
\end{aligned}
$$

Let $t_{m}, t_{k} \in\left(0, T^{*}\right)$ be any two sequences satisfying $\lim _{m \rightarrow \infty} t_{m}=\lim _{k \rightarrow \infty} t_{k}=T^{*}$. Then for any $j=1,2, \ldots, N$, we have

$$
\left.\left|\int_{\mathbb{R}^{N}}\right| u\left(t_{m}, x\right)\right|^{2} x_{j} d x-\int_{\mathbb{R}^{N}}\left|u\left(t_{k}, x\right)\right|^{2} x_{j} d x|\leq C| t_{m}-t_{k} \mid \rightarrow 0 \text { as } m, k \rightarrow \infty,
$$

which implies that

$$
\lim _{t \rightarrow T^{*}} \int_{\mathbb{R}^{N}}|u(t, x)|^{2} x_{j} d x \text { exists for any } j=1,2, \ldots, N \text {. }
$$

Set

$$
x_{0}=\|Q\|_{L^{2}}^{-2} \lim _{t \rightarrow T^{*}} \int_{\mathbb{R}^{N}}|u(t, x)|^{2} x d x .
$$


Then

$$
\lim _{t \rightarrow T^{*}} \int_{\mathbb{R}^{N}}|u(t, x)|^{2} x d x=x_{0}\|Q\|_{L^{2}}^{2} .
$$

On the other hand, we infer from Lemma 2.2 that there is a constant $c_{0}$ such that

$$
\int_{\mathbb{R}^{N}}|x|^{2}|u(t, x)|^{2} d x \leq c_{0}
$$

This yields

$$
\begin{aligned}
\int_{\mathbb{R}^{N}}|x|^{2}|u(t, x+x(t))|^{2} d x & \leq 2 \int_{\mathbb{R}^{N}}|x+x(t)|^{2}|u(t, x+x(t))|^{2} d x+2|x(t)|^{2} \int_{\mathbb{R}^{N}}|u(t, x+x(t))|^{2} d x \\
& \leq 2 c_{0}+2|x(t)|^{2}\left\|u_{0}\right\|_{L^{2}}^{2}
\end{aligned}
$$

We infer from (4.15) that

$$
\limsup _{t \rightarrow T^{*}}|x(t)|^{2}\|Q\|_{L^{2}}^{2}=\limsup _{t \rightarrow T^{*}} \int_{|x|<1}|x+x(t)|^{2}|u(t, x+x(t))|^{2} d x \leq \int_{\mathbb{R}^{N}}|x|^{2}|u(t, x)|^{2} d x \leq c_{0} .
$$

Thus,

$$
\limsup _{t \rightarrow T^{*}}|x(t)| \leq \frac{\sqrt{c_{0}}}{\|Q\|_{L^{2}}}
$$

Combining (4.18) and (4.19), we obtain

$$
\limsup _{t \rightarrow T^{*}} \int_{\mathbb{R}^{N}}|x|^{2}|u(t, x+x(t))|^{2} d x \leq C
$$

Hence, for any $\varepsilon>0$, there exists $R_{0}=R_{0}(\varepsilon)$ such that

$$
\left.\limsup _{t \rightarrow T^{*}}\left|\int_{|x| \geq R_{0}} x\right| u(t, x+x(t))\right|^{2} d x \mid \leq \frac{C}{R_{0}}<\varepsilon .
$$

It follows from (4.15) that

$$
\begin{aligned}
\left.\limsup _{t \rightarrow T^{*}}\left|\int_{\mathbb{R}^{N}}\right| u(t, x)\right|^{2} x d x-x(t)\|Q\|_{L^{2}}^{2} \mid & =\left.\limsup _{t \rightarrow T^{*}}\left|\int_{\mathbb{R}^{N}}\right| u(t, x)\right|^{2}(x-x(t)) d x \mid \\
& \leq\left.\limsup _{t \rightarrow T^{*}}\left|\int_{|x| \leq R_{0}}\right| u(t, x+x(t))\right|^{2} x d x \mid+\varepsilon \\
& \leq \varepsilon
\end{aligned}
$$

which, together with (4.17) implies that $\lim _{t \rightarrow T^{*}} x(t)=x_{0}$. Therefore,

$$
\limsup _{t \rightarrow T^{*}} \int_{\mathbb{R}^{N}}|u(t, x)|^{2} x d x=\|Q\|_{L^{2}}^{2} x_{0}
$$


and

$$
|u(t, x)|^{2} \rightarrow\|Q\|_{L^{2}}^{2} \delta_{x=x_{0}} \text { in the sence of distribution as } t \rightarrow T^{*} \text {. }
$$

The following theorem gives the lower bound for the blow-up rate of blow-up solutions with critical mass $\left\|u_{0}\right\|_{L^{2}}=\|Q\|_{L^{2}}$.

Theorem 4.5. Let $u_{0} \in \Sigma, \lambda_{1}=-1, \lambda_{2}=1, p_{1}=\frac{4}{N}$, and $0<p_{2}<\frac{4}{N}$. If the solution $u$ of (1.2) blows up in finite time $T^{*}>0$ and $\left\|u_{0}\right\|_{L^{2}}=\|Q\|_{L^{2}}$, then there exists a constant $C>0$ such that

$$
\|\nabla u(t)\|_{L^{2}} \geq \frac{C}{T^{*}-t}, \quad \forall t \in\left[0, T^{*}\right)
$$

Proof. Let $h \in C_{0}^{\infty}\left(\mathbb{R}^{N}\right)$ be a nonnegative radial function such that

$$
h(x)=h(|x|)=|x|^{2} \text {, if }|x|<1 \text { and }|\nabla h(x)|^{2} \leq C h(x) .
$$

For $A>0$, we define $h_{A}(x)=A^{2} h\left(\frac{x}{A}\right)$ and $g_{A}(t)=\int h_{A}\left(x-x_{0}\right)|u(t, x)|^{2} d x$ with $x_{0}$ defined by (41).

From (4.16), for every $t \in\left[0, T^{*}\right)$, we have

$$
\begin{aligned}
\left|\frac{d}{d t} g_{A}(t)\right| & =2\left|\operatorname{Im} \sum_{j=1}^{N} \int_{\mathbb{R}^{N}} \bar{u}(t, x) \nabla u(t, x) \nabla h_{A}\left(x-x_{0}\right) d x\right| \\
& \leq 2 \sqrt{E\left(u_{0}\right)}\left(\int_{\mathbb{R}^{N}}|u(t, x)|^{2}\left|\nabla h_{A}\left(x-x_{0}\right)\right|^{2} d x\right)^{1 / 2} \\
& \leq C \sqrt{g_{A}(t)}
\end{aligned}
$$

which implies

$$
\left|\frac{d}{d t} \sqrt{g_{A}(t)}\right| \leq C .
$$

Integrating on both sides, we obtain

$$
\left|\sqrt{g_{A}(t)}-\sqrt{g_{A}\left(t_{1}\right)}\right| \leq C\left|t-t_{1}\right|
$$

It follows from (4.13) that

$$
g_{A}\left(t_{1}\right) \rightarrow\|Q\|_{L^{2}} h_{A}(0)=0 \quad \text { as } t_{1} \rightarrow T^{*} .
$$


Therefore, letting $t_{1} \rightarrow T^{*}$ in (4.23), it follows that

$$
g_{A}(t) \leq C\left(T^{*}-t\right)^{2}
$$

Now fix $t \in\left[0, T^{*}\right)$ and let $A$ go to infinity, we have

$$
\int_{\mathbb{R}^{N}}\left|x-x_{0}\right|^{2}|u(t, x)|^{2} d x \leq C\left(T^{*}-t\right)^{2} .
$$

Then the uncertainty principle

$$
\left(\int_{\mathbb{R}^{N}}|u(t, x)|^{2} d x\right)^{2} \leq\left(\int_{\mathbb{R}^{N}}\left|x-x_{0}\right|^{2}|u(t, x)|^{2} d x\right)\left(\int_{\mathbb{R}^{N}}|\nabla u(t, x)|^{2} d x\right),
$$

implies a lower bound of the blow-up rate

$$
\|\nabla u(t)\|_{L^{2}} \geq \frac{C}{T^{*}-t}, \quad \forall t \in\left[0, T^{*}\right) .
$$

\section{The $L^{2}$-supercritical case}

When $\lambda_{1}<0, \lambda_{2} \in \mathbb{R}, \frac{4}{N}<p_{1}<\frac{4}{N-2}$ and $0<p_{2}<p_{1}$, for some large initial data, the solution may blow up in finite time. In order to investigate some concentration properties of the blow-up solutions to (1.2) in this case, we need the following version of compactness lemma which comes from [9].

Lemma 5.1. Let $\left\{u_{n}\right\}_{n=1}^{\infty}$ be a bounded sequence in $\dot{H}^{s_{c}} \cap \dot{H}^{1}$, such that

$$
\limsup _{n \rightarrow \infty}\left\|\nabla u_{n}\right\|_{L^{2}} \leq M, \quad \limsup _{n \rightarrow \infty}\left\|u_{n}\right\|_{L^{p_{1}+2}} \geq m>0 .
$$

Then, there exist $\left\{x_{n}^{1}\right\}_{n=1}^{\infty},\left\{x_{n}^{2}\right\}_{n=1}^{\infty} \subset \mathbb{R}^{N}, V_{1} \in \dot{H}^{s_{c}} \cap \dot{H}^{1}$, and $V_{2} \in L^{p_{c}} \cap \dot{H}^{1}$ such that, up to a subsequence,

$$
u_{n}\left(\cdot+x_{n}^{1}\right) \rightarrow V_{1} \text { weakly in } \dot{H}^{s_{c}} \cap \dot{H}^{1}
$$

with

$$
\left\|V_{1}\right\|_{\dot{H}^{s_{c}}}^{p_{1}} \geq \frac{2 m^{p_{1}+2}}{\left(p_{1}+2\right) M^{2}}\|Q\|_{\dot{H}^{s_{c}}}^{p_{1}}
$$


and

$$
u_{n}\left(\cdot+x_{n}^{2}\right) \rightarrow V_{2} \text { weakly in } L^{p_{c}} \cap \dot{H}^{1},
$$

with

$$
\left\|V_{2}\right\|_{L^{p_{c}}}^{2} \geq \frac{2 m^{p_{1}+2}}{\left(p_{1}+2\right) M^{2}}\|R\|_{L^{p_{c}}}^{2}
$$

where $Q$ and $R$ are the solutions of the following elliptic equations

$$
-\Delta Q+\frac{p_{1}}{2}(-\triangle)^{s_{c}} Q=|Q|^{p_{1}} Q
$$

and

$$
-\Delta R+|R|^{p_{c}-2} R=|R|^{p_{1}} R
$$

respectively.

In the following theorem, we will use the ground states of equations (5.1) and (5.2) to describe some concentration properties of blow-up solutions to (1.2). This result is closed related to the result obtained for the classical nonlinear Schrödinger equation (1.1) with $\frac{4}{N}<p<\frac{4}{N-2}$ by Guo [9].

Theorem 5.2. Let $\lambda_{1}<0, \lambda_{2} \in \mathbb{R}, \frac{4}{N}<p_{1}<\frac{4}{N-2}, 0<p_{2}<p_{1}$ and $u_{0} \in \dot{H}^{s_{c}} \cap \dot{H}^{1}$. If the solution $u$ of (1.2) blows up in finite time $T^{*}>0$ and satisfies

$$
\sup _{t \in\left[0, T^{*}\right)}\|u(t)\|_{\dot{H}^{s_{c}}}<\infty
$$

Assume that $\lambda(t)>0$ such that

$$
\lambda(t)\|\nabla u(t)\|_{L^{2}}^{\frac{1}{s_{c}}} \rightarrow \infty
$$

as $t \rightarrow T^{*}$. Then, there exist $x_{1}(t), x_{2}(t) \in \mathbb{R}^{N}$ such that

$$
\liminf _{t \rightarrow T^{*}} \int_{\left|x-x_{1}(t)\right| \leq \lambda(t)}\left|(-\triangle)^{\frac{s_{c}}{2}} u(t, x)\right|^{2} d x \geq\|Q\|_{\dot{H}^{s_{c}}}^{2},
$$

and

$$
\liminf _{t \rightarrow T^{*}} \int_{\left|x-x_{2}(t)\right| \leq \lambda(t)}|u(t, x)|^{p_{c}} d x \geq\|R\|_{L^{p_{c}}}^{p_{c}},
$$

where $Q$ and $R$ solve the elliptic equations (5.1) and (5.2), respectively. 
Proof. Set

$$
\rho(t)=\|\nabla Q\|_{L^{2}}^{\frac{1}{1-s_{c}}} /\|\nabla u(t)\|_{L^{2}}^{\frac{1}{1-s_{c}}} \text { and } v(t, x)=\rho^{\frac{2}{p_{1}}}(t) u(t, \rho(t) x) .
$$

Let $\left\{t_{n}\right\}_{n=1}^{\infty}$ be an any time sequence such that $t_{n} \rightarrow T^{*}, \rho_{n}=\rho\left(t_{n}\right)$ and $v_{n}(x)=v\left(t_{n}, x\right)$. Then, it follows from assumption (5.3) that $v_{n}$ satisfies $\left\|v_{n}\right\|_{\dot{H}^{s_{c}}}=\left\|u\left(t_{n}\right)\right\|_{\dot{H}^{s_{c}}}<\infty$ uniformly in $n$. Moreover, by some direct computations, we obtain

$$
\left\|\nabla v_{n}\right\|_{L^{2}}=\rho_{n}^{1-s_{c}}\left\|\nabla u\left(t_{n}\right)\right\|_{L^{2}}=\|\nabla Q\|_{L^{2}}
$$

and

$$
\begin{aligned}
H\left(v_{n}\right) & :=\frac{1}{2} \int_{\mathbb{R}^{N}}\left|\nabla v_{n}(x)\right|^{2} d x+\frac{\lambda_{1}}{p_{1}+2} \int_{\mathbb{R}^{N}}\left|v_{n}(x)\right|^{p_{1}+2} d x \\
& =\rho_{n}^{2\left(1-s_{c}\right)}\left(\frac{1}{2} \int_{\mathbb{R}^{N}}\left|\nabla u\left(t_{n}, x\right)\right|^{2} d x+\frac{\lambda_{1}}{p_{1}+2} \int_{\mathbb{R}^{N}}\left|u\left(t_{n}, x\right)\right|^{p_{1}+2} d x\right) \\
& =\rho_{n}^{2\left(1-s_{c}\right)}\left(E\left(u\left(t_{n}\right)\right)-\frac{\lambda_{2}}{p_{2}+2} \int_{\mathbb{R}^{N}}\left|u\left(t_{n}, x\right)\right|^{p_{2}+2} d x\right) \\
& =\frac{\|\nabla Q\|_{L^{2}}^{2}}{\left\|\nabla u\left(t_{n}\right)\right\|_{L^{2}}^{2}}\left(E\left(u_{0}\right)-\frac{\lambda_{2}}{p_{2}+2} \int_{\mathbb{R}^{N}}\left|u\left(t_{n}, x\right)\right|^{p_{2}+2} d x\right) .
\end{aligned}
$$

In the following, we will prove $H\left(v_{n}\right) \rightarrow 0$ as $n \rightarrow \infty$, which implies $\int_{\mathbb{R}^{N}}\left|v_{n}(x)\right|^{p+2} d x \rightarrow$ $2\|\nabla Q\|_{L^{2}}^{2}$.

When $0<p_{2}<\frac{4}{N}$, note that the following Gagliardo-Nirenberg inequality

$$
\int_{\mathbb{R}^{N}}|u(x)|^{p_{2}+2} d x \leq C\|u\|_{L^{2}}^{\frac{2\left(p_{2}+2\right)-N p_{2}}{2}}\|\nabla u\|_{L^{2}}^{\frac{N p_{2}}{2}} .
$$

By the similar argument as (4.4), it follows that $H\left(v_{n}\right) \rightarrow 0$ as $n \rightarrow \infty$.

When $\frac{4}{N} \leq p_{2}<p_{1}<\frac{4}{N-2}$ and $\frac{N p_{1}}{2}<p_{2}+2$, we can obtain the following GagliardoNirenberg inequality

$$
\int_{\mathbb{R}^{N}}|u(x)|^{p_{2}+2} d x \leq C\|u\|_{L^{\frac{N p_{1}}{2}}}^{\left(p_{2}+2\right)\left(1-\theta_{1}\right)}\|\nabla u\|_{L^{2}}^{\theta_{1}\left(p_{2}+2\right)},
$$

where $\theta_{1}=\frac{4 p_{2}+8-2 N p_{1}}{\left(p_{2}+2\right)\left(2 p_{1}+4-N p_{1}\right)}$. Thus, we deduce from $\theta_{1}\left(p_{2}+2\right)<2$ that $H\left(v_{n}\right) \rightarrow 0$ as $n \rightarrow \infty$.

When $\frac{4}{N} \leq p_{2}<p_{1}<\frac{4}{N-2}$ and $\frac{N p_{1}}{2}>p_{2}+2$, we have

$$
\int_{\mathbb{R}^{N}}|u(x)|^{p_{2}+2} d x \leq C\|u\|_{L^{2}}^{\left(1-\theta_{2}\right)\left(p_{2}+2\right)}\|u\|_{L^{\frac{N p_{1}}{2}}}^{\theta_{2}\left(p_{2}+2\right)} \leq C\|u\|_{L^{2}}^{\left(1-\theta_{2}\right)\left(p_{2}+2\right)}\|u\|_{H^{s_{c}}}^{\theta_{2}\left(p_{2}+2\right)},
$$

where $\theta_{2}=\frac{N p_{1} p_{2}}{\left(p_{2}+2\right)\left(N p_{1}-4\right)}$. This inequality and the assumption (5.3) imply that $H\left(v_{n}\right) \rightarrow 0$ as $n \rightarrow \infty$. 
When $\frac{4}{N} \leq p_{2}<p_{1}<\frac{4}{N-2}$ and $\frac{N p_{1}}{2}=p_{2}+2$, it follows from the Sobolev embedding that

$$
\int_{\mathbb{R}^{N}}|u(x)|^{p_{2}+2} d x=\|u\|_{L^{\frac{N p_{1}}{2}}}^{p_{2}+2} \leq C\|u\|_{H^{s_{c}}}^{p_{2}+2},
$$

which, together with the assumption (5.3), implies that $H\left(v_{n}\right) \rightarrow 0$ as $n \rightarrow \infty$.

Set $m=2\|\nabla Q\|_{L^{2}}^{2}$ and $M=\|\nabla Q\|_{L^{2}}^{2}$. Then it follows from Lemma 4.1 that there exist $V \in \dot{H}^{s_{c}} \cap \dot{H}^{1}$ and $\left\{x_{n}\right\}_{n=1}^{\infty} \subset \mathbb{R}^{N}$ such that, up to a subsequence,

$$
v_{n}\left(\cdot+x_{n}\right)=\rho_{n} u\left(t_{n}, \rho_{n} \cdot+x_{n}\right) \rightarrow V \text { weakly in } \dot{H}^{s_{c}} \cap \dot{H}^{1}
$$

with

$$
\|V\|_{\dot{H}^{s_{c}}}^{2} \geq \frac{m}{2 M}\|Q\|_{\dot{H}^{s_{c}}}^{2}
$$

By the definition of $\dot{H}^{s_{c}}$, we have

$$
(-\Delta)^{\frac{s_{c}}{2}} \rho_{n} u\left(t_{n}, \rho_{n} \cdot+x_{n}\right) \rightarrow(-\Delta)^{\frac{s_{c}}{2}} V \text { weakly in } L^{2}
$$

Thus, for any $R>0$,

$$
\int_{|x| \leq R}\left|(-\Delta)^{\frac{s_{c}}{2}} V(x)\right|^{2} d x \leq \liminf _{n \rightarrow \infty} \int_{\left|x-x_{n}\right| \leq \rho_{n} R}\left|(-\Delta)^{\frac{s_{c}}{2}} u\left(t_{n}, x\right)\right|^{2} d x
$$

In view of the assumption $\lambda\left(t_{n}\right) / \rho_{n} \rightarrow \infty$, this implies immediately

$$
\int_{|x| \leq R}\left|(-\Delta)^{\frac{s_{c}}{2}} V\right|^{2} d x \leq \liminf _{n \rightarrow \infty} \sup _{y \in \mathbb{R}^{N}} \int_{|x-y| \leq \lambda\left(t_{n}\right)}\left|(-\Delta)^{\frac{s_{c}}{2}} u\left(t_{n}, x\right)\right|^{2} d x
$$

Since the sequence $\left\{t_{n}\right\}_{n=1}^{\infty}$ is arbitrary, we obtain

$$
\int_{|x| \leq R}\left|(-\Delta)^{\frac{s_{c}}{2}} V\right|^{2} d x \leq \liminf _{n \rightarrow \infty} \sup _{y \in \mathbb{R}^{N}} \int_{|x-y| \leq \lambda(t)}\left|(-\Delta)^{\frac{s_{c}}{2}} u(t, x)\right|^{2} d x .
$$

Observe that for every $t \in[0, T)$, the function $y \mapsto \int_{|x-y| \leq \lambda(t)}\left|(-\Delta)^{\frac{s_{c}}{2}} u(t, x)\right|^{2} d x$ is continuous and goes to zero at infinity. Thus, there exists $x(t) \in \mathbb{R}^{N}$ such that

$$
\int_{|x-x(t)| \leq \lambda(t)}\left|(-\Delta)^{\frac{s_{c}}{2}} u(t, x)\right|^{2} d x=\sup _{y \in \mathbb{R}^{N}} \int_{|x-y| \leq \lambda(t)}\left|(-\Delta)^{\frac{s_{c}}{2}} u(t, x)\right|^{2} d x .
$$

This and (5.12) yield (5.5). The proof of (5.6) is similar, so we omit it. This completes the proof. 


\section{References}

[1] T. Cazenave, Semilinear Schrödinger equations, Courant Lecture Notes in Mathematics vol. 10, New York University, Courant Institute of Mathematical Sciences, New York; American Mathematical Society, Providence, RI, 2003.

[2] B. Feng, Sharp threshold of global existence and instability of standing wave for the SchrödingerHartree equation with a harmonic potential, Nonlinear Anal. Real World Appl., 31(2016) 132-145.

[3] B. Feng, Y. Cai, Concentration for blow-up solutions of the Davey-Stewartson system in $\mathbb{R}^{3}$, Nonlinear Anal. Real World Appl., 26(2015) 330-342.

[4] B. Feng, X. Yuan, On the Cauchy problem for the Schrödinger-Hartree equation, Evol. Equ. Control Theory, 4(2015) 431-445.

[5] B. Feng, D. Zhao, C. Sun, On the Cauchy problem for the nonlinear Schrödinger equations with time-dependent linear loss/gain, J. Math. Anal. Appl., 416(2014) 901-923.

[6] G. Fibich, The Nonlinear Schrödinger Equation, Singular Solutions and Optical Collapse, Springer, 2015.

[7] J. Ginibre, G. Velo, On a class of nonlinear Schrödinger equations. I. The Cauchy problem, general case, J. Funct. Anal., 32(1979) 1-32.

[8] R.T. Glassey, On the blowing up of solutions to the Cauchy problem for nonlinear Schrödinger equations, J. Math. Phys., 18(1977) 1794-1797.

[9] Q. Guo, A note on concentration for blowup solutions to supercritical Schrödinger equations, Proc. Amer. Math. Soc., 141(2013) 4215-4227.

[10] T. Hmidi, S. Keraani, Blowup theory for the critical nonlinear Schrödinger equations revisited, International Mathematics Research Notices, 46(2005) 2815-2828.

[11] X. Li, J. Zhang, S. Y. Lai, Y. Wu, The sharp threshold and limiting profile of blow-up solutions for a Davey-Stewartson system, J. Diff. Eqns., 250(2011) 2197-2226.

[12] F. Merle, P. Raphaël, On universality of blow-up profile for $L^{2}$ critical nonlinear Schrödinger equation, Invent. Math., 156(2004) 565-572.

[13] F. Merle, P. Raphaël, Blow-up dynamic and upper bound on the blow-up rate for critical nonlinear Schrödinger equation, Ann. Math., 16(2005) 157-222.

[14] F. Merle, P. Raphaël, On a sharp lower bound on the blow-up rate for the $L^{2}$ critical nonlinear Schrödinger equation, J. Amer. Soc., 19(2006) 37-90.

[15] F. Merle, P. Raphaël, Profiles and quantization of the blowup mass for critical nonlinear Schrödinger equation, Comm. Math. Phys., 253(2005) 675-704. 
[16] B. V. Sz. Nagy, Uber Integralgleichungen zwischen einer Funktion und ihrer Ableitung, Acta Sci. Math., 10(1941) 64-74.

[17] T. Ogawa, Y. Tsutsumi, Blow-up of $H^{1}$ solution for the nonlinear Schrödinger equation, J. Differential Equations, 92 (1991) 317-330.

[18] C. Sulem, P.L. Sulem, The nonlinear Schrödinger equation, Applied Math. Sciences 139, Springer 1999.

[19] T. Tao, Nonlinear Dispersive Equations: Local and Global Analysis, CBMSReg. Conf. Ser. Math., AMS, 2006.

[20] T. Tao, M. Visan, X. Zhang, The nonlinear Schrödinger equation with combined power-type nonlinearities, Comm. Partial Differential Equations, 32(2007) 1281-1343.

[21] M.I. Weinstein, Nonlinear Schrödinger equations and sharp interpolation estimates, Comm. Math. Phys., 87(1983) 567-576.

[22] M.I. Weinstein, On the structure and formation of singularities in solutions to nonlinear dispersive evolution equa-tions, Comm. Partial Differential Equations, 11(1986) 545-565.

[23] J. Zhang, Sharp conditions of global existence for nonlinear Schrödinger and Klein-Gordon equations, Nonlinear Anal., 48(2002) 191-207.

[24] J. Zhang, S. Zhu, Sharp blow-up criteria for the Davey-Stewartson system in $\mathbb{R}^{3}$, Dynamics of PDE, 8(2011) 239-260.

[25] S. Zhu, On the blow-up solutions for the nonlinear fractional Schrödinger equation, J. Differential Equations, 261(2016) 1506-1531.

[26] S. Zhu, On the Davey-Stewartson system with competing nonlinearities, J. Math. Phys., 57(2016) 031501.

[27] S. Zhu, Blow-up solutions for the inhomogeneous Schrödinger equation with $L^{2}$ supercritical nonlinearity, J. Math. Anal. Appl., 409(2014) 760-776. 